INTERNATIONAL JOURNAL
PHARMACEUTICAL SCIENCES
RESEARCH
PHPI

Received on 10 July 2020; received in revised form, 09 December 2020; accepted, 15 May 2021; published 01 July 2021

\title{
RP-HPLC METHOD DEVELOPMENT AND VALIDATION FOR THE SIMULTANEOUS ESTIMATION OF LEDIPASVIR AND SOFOSBUVIR IN FIXED DOSAGE FORM
}

\author{
V. Pavan Kumar ${ }^{1}$, Narayanaswamy Harikrishnan ${ }^{*} 2$ and Gejalakshmi Subramanian ${ }^{2}$
}

Department of Pharmaceutical Analysis ${ }^{1}$, Seven Hills College of Pharmacy, Venkatramapuram, Tirupati517561, Andhra Pradesh, India.

Department of Pharmaceutical Analysis ${ }^{2}$, Faculty of Pharmacy, Dr. M. G. R. Educational and Research Institute, Velappanchavadi, Chennai - 600077, Tamil Nadu, India.

Keywords:

Sofosbuvir, Ledipasvir, RP-HPLC, Quality Control, Validation

\section{Correspondence to Author:}

Dr. N. Harikrishnan

Principal,

Faculty of Pharmacy, Dr. M.G.R.

Educational and Research Institute,

Velappanchavadi, Chennai - 600077,

Tamil Nadu, India.

E-mail: pavanvarikuti87@gmail.com
ABSTRACT: Ledipasvir and Sofosbuvir Combination have been approved for the treatment of Chronic Hepatitis C Viral Infection. Here an accurate, valid, elementary, and error-free reverse-phase liquid chromatography strategy was developed for the quantitation of Ledipasvir and Sofosbuvir in its bulk form as well as in fixed dosage form. Effective chromatographic separation of Ledipasvir and Sofosbuvir was achieved by using Kromasil C$18(250 \times 4.6 \mathrm{~mm}, 5 \mu \mathrm{m})$ column using Phosphate buffer $(\mathrm{pH} \mathrm{3.5)}$ and Methanol in the proportion of 45:55 v/v. The Mobile phase was siphoned at a flow rate of $1.0 \mathrm{~mL} \mathrm{~min}{ }^{-1}$ with a column temperature of $35^{\circ} \mathrm{C}$, and detection wavelength was carried out at $259 \mathrm{~nm}$. The retention time was found to be $3.294 \mathrm{~min}$ for Sofosbuvir and $4.630 \mathrm{~min}$ for Ledipasvir. The dimensionality of Sofosbuvir and Ledipasvir was in linear range with a parametric static of 0.999 and 0.999. Method Validation was carried out in terms of Specificity, Linearity, Precision, Accuracy, LOD, LOQ as per ICH Guidelines. Results obtained from the validation studies show that the developed method can be useful in the quality control analysis of bulk and pharmaceutical formulations of Ledipasvir and Sofosbuvir.
INTRODUCTION: Chronic Hepatitis $\mathrm{C}$ affects a large number of people worldwide ${ }^{1}$. It is a viral infection that attacks the liver and leads to inflammation $^{2}$. Chronic Hepatitis $\mathrm{C}$ treatment has continued to evolve and interferon-free, oral treatment with a combination of Sofosbuvir and Ledipasvir, which are two direct-acting anti-viral agents ${ }^{3}$. The Oral administration of Sofosbuvir and Ledipasvir combination was well tolerated and suppressed the effect of predictive factors of Chronic Hepatitis ${ }^{4}$.

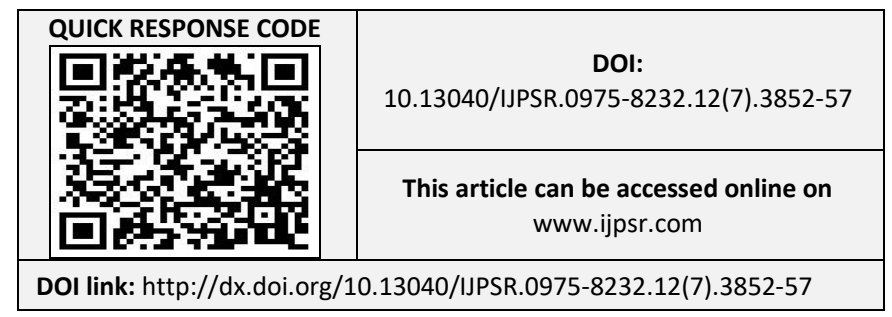

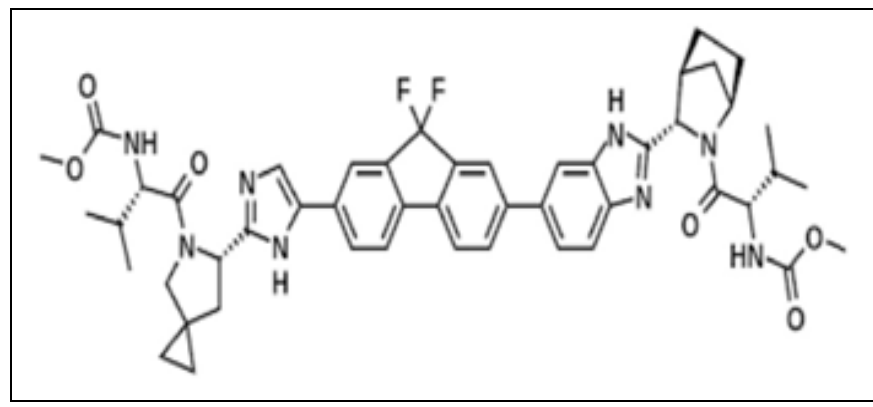

FIG. 1: CHEMICAL STRUCTURE OF LEDIPASVIR

Ledipasvir is anti-viral drug chemically (2S)-1[(6S)-6-[5-(9, 9-difluoro-7-\{2-[1R, 3S, 4S)-2-[(2S)2-\{[hydroxyl(methoxy)methydene] amino $\}-3-$ methylbutanoyl]-2-azabicyclo[2.2.1]heptan-3-yl]-1H-1, 3benzodiazol-6-yl $-9 H-F l u o r e n-2-y l)-1 \mathrm{H}$-imidazole2- yl]- 5- azaspirol[2.4]heptan- 5- yl]-2 \{[hydroxyl (methoxy)methylidene]amino - 3- methylbutan- 1one having formula $\mathrm{C}_{49} \mathrm{H}_{54} \mathrm{~F}_{2} \mathrm{~N}_{8} \mathrm{O}_{6}$ and relative 
molecular mass of $889.00 \mathrm{~g} / \mathrm{mol}{ }^{5}$. It acts by inhibiting NS5A protein which is mainly responsible for viral RNA Replication ${ }^{6}$. The chemical structure of Ledipasvir is exhibited in Fig. 1.

Sofosbuvir is [1-4] isopropyl(2S)-2-[[[(2R,3R,4R, 5R)- 5- (2, 4- dioxoprrimidin- 1- yl)- 4-fluoro-3hydroxy- 4- methyl-tetrahydrofuran-2-yl]methoxyphenoxyphosphoryl] amino] propanoate having formula $\mathrm{C}_{22} \mathrm{H}_{29} \mathrm{FN}_{3} \mathrm{O}_{9} \mathrm{P}$ and relative molecular mass of $529.45 \mathrm{~g} / \mathrm{mol}^{5}$. It acts by inhibiting NS5B polymerase used in the treatment of hepatitis $C^{7}$. The chemical structure of Sofosbuvir is exhibited in Fig. 2.

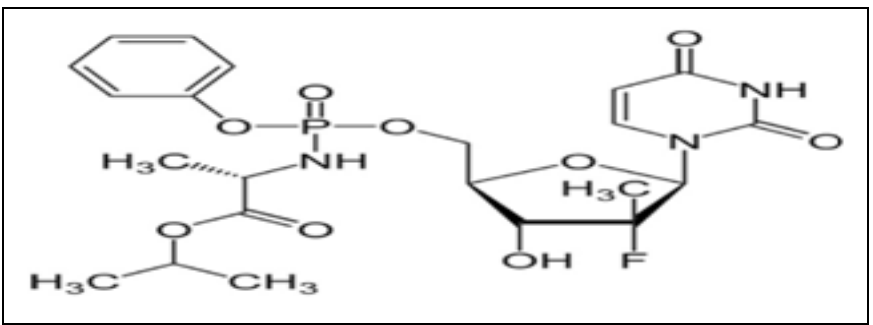

FIG. 2: CHEMICAL STRUCTURE OF SOFOSBUVIR

The pharmaceutical dosage form having a combination of Ledipasvir and Sofosbuvir provides a new method of treatment effectively for several people suffering from chronic hepatitis C Virus Infection ${ }^{8}$. The present strategy focused on isocratic high-performance liquid chromatography method for the estimation of Ledipasvir and Sofosbuvir. After performing an extensive literature review, an attempt was made to develop a smooth plain sailing, unambiguous, valid, speedy, and decisive strategy for estimating Ledipasvir and Sofosbuvir in fixed dosage form ${ }^{9-13}$.

\section{MATERIALS AND METHODS:}

Chemicals and Reagents: Ledipasvir and Sofosbuvir were obtained as a gift sample from Nutech Biosciences Pvt., Ltd., Hyderabad, India, certified to contain acceptable purity limit and were used without any refinement. HPLC grade solvents were used in chromatographic separation of Ledipasvir and Sofosbuvir, and a $0.45 \mu$ membrane filter was obtained from Millipore. Ledifos Tablets (label claim $90 \mathrm{mg}$ of Ledipasvir and $400 \mathrm{mg}$ of Sofosbuvir) of Hetero Health care obtained from the local pharmacy were used in the analysis

Instrument: The liquid chromatography system used was Waters Alliance having to empower software for processing the data with a 2695 separation module equipped with a PDA detector with a universal loop injector of injection capacity $20 \mu \mathrm{l}$. The analytical column that was selected for ideal separation was the Kromasil C-18 $(250 \times 4.6$ $\mathrm{mm}$, packed with a particle size of $5 \mu \mathrm{m}$ ) column. Several solvents in different proportions were tested in order to determine the suitable conditions for the separation of drugs.

Optimized Chromatographic conditions: The mobile phase selected was a mixture of phosphate buffer ( $\mathrm{pH} 3.5)$ and Methanol in the proportion of $45: 55 \% \mathrm{v} / \mathrm{v}$ at a flow rate of $1.0 \mathrm{~mL} / \mathrm{min}$ as it resolves the height with retention times of 3.294 min and $4.630 \mathrm{~min}$ for Ledipasvir respectively. Standard drug solutions were scanned over a range from 200 to $400 \mathrm{~nm}$, and detection was carried out at $259 \mathrm{~nm}$ as both the drugs showed reasonably good response with characteristic UV spectrum as exhibited in Fig. 3.

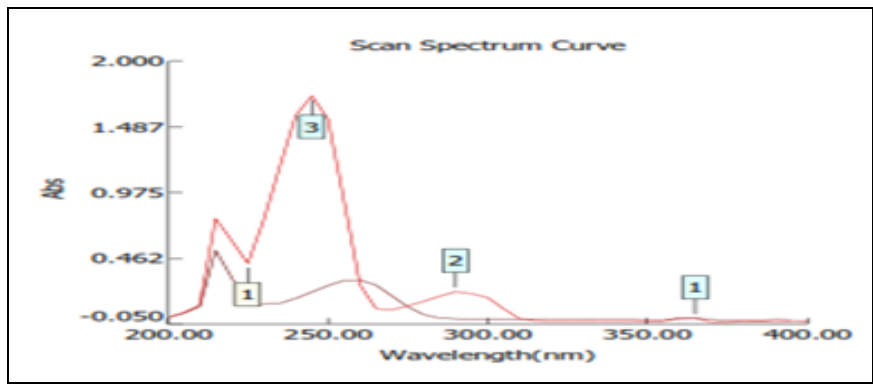

FIG. 3: ISOBESTIC POINT OF LEDIPASVIR AND SOFOSBUVIR

Buffer Preparation: Accurately weighed and transferred a quantity equivalent to $1.732 \mathrm{~g}$ of Potassium Dihydrogen Orthophosphate into a 500 $\mathrm{ml}$ clean and dried volumetric flask. Into the above volumetric flask, $500 \mathrm{ml}$ of HPLC water was added and subjected to sonication for three minutes to dissolve phosphate buffer completely, and the volume was made up to the mark with the same solvent, and $\mathrm{pH}$ was adjusted to 3.5 by adding few drops of Orthophosphoric acid.

Mobile Phase Preparation: Accurately measured 450 milliliter of Phosphate buffer $(\mathrm{pH} \mathrm{3.5)} \mathrm{(45 \% )}$ and $550 \mathrm{ml}$ of Methanol $(55 \%)$ were mixed and subjected to sonication in an inaudible water tub for five minutes, and after sonication, the mobile phase was filtered using $0.45 \mu$ membrane filter under vacuum before its use 
Diluent: Mobile phase was used as diluent.

Preparation of Standard Solution: Accurately weighed and transferred a quantity which is equivalent to $6 \mathrm{mg}$ of Ledipasvir and $15 \mathrm{mg}$ of Sofosbuvir working standard into a $10 \mathrm{ml}$ clean and dry volumetric flask and add $7 \mathrm{ml}$ of diluent. The above solution was sonicated for few minutes until the drug dissolves, and volume was made up to the mark with the same solvent. Further pipette out 1 $\mathrm{ml}$ of the above stock solutions into a $10 \mathrm{ml}$ volumetric flask and dilute up to the mark with diluent. The chromatogram of the standard solution was exhibited in Fig. 4.

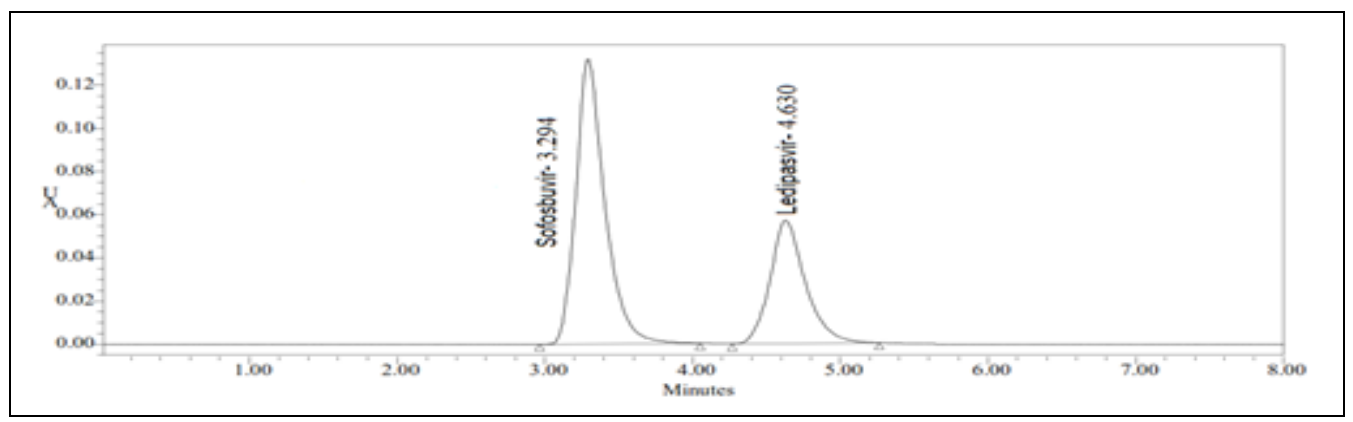

FIG. 4: STANDARD CHROMATOGRAM OF LEDIPASVIR AND SOFOSBUVIR

Preparation of Sample Solution: Accurately, 10 tablets were taken and crushed in mortar and pestle and transferred an amount equivalent to $6 \mathrm{mg}$ of Ledipasvir and $15 \mathrm{mg}$ of Sofosbuvir sample into a $10 \mathrm{ml}$ clean, dry volumetric flask and add $7 \mathrm{ml}$ of diluent. The above solution was sonicated for few minutes until the drug dissolves, and volume was made up to the mark with the same solvent. Further pipette out $1 \mathrm{ml}$ of the above stock solutions into a $10 \mathrm{ml}$ volumetric flask and dilute up to the mark with diluent. Inject $20 \mu \mathrm{L}$ of the standard sample into the chromatographic system and measure the peak areas for Sofosbuvir and Ledipasvir and calculate the \% Assay by using the formulae. The chromatogram of the sample solution was exhibited in Fig. 5.

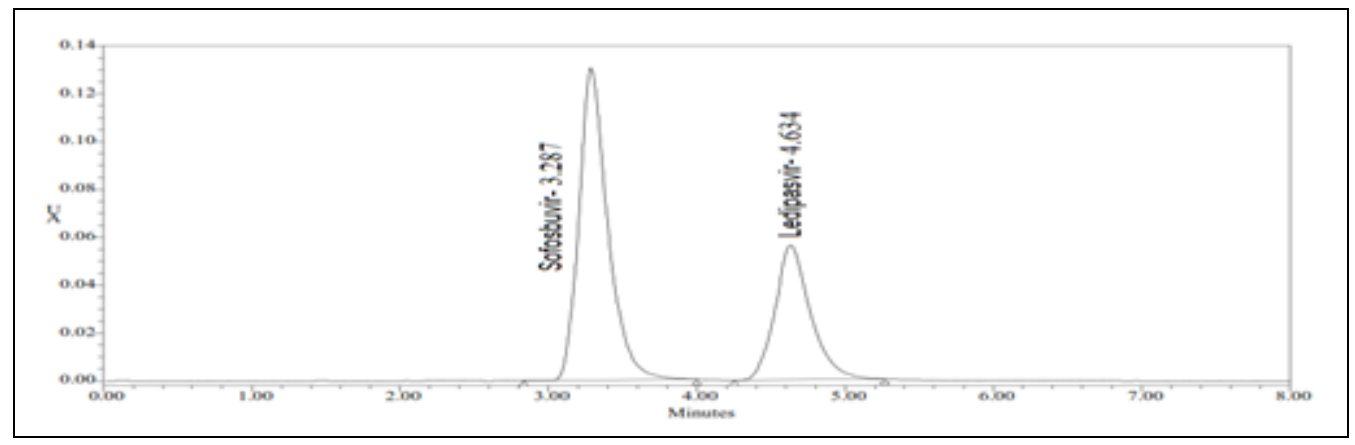

FIG. 5: SAMPLE CHROMATOGRAM OF LEDIPASVIR AND SOFOSBUVIR

\section{RESULTS AND DISCUSSION:}

Method Validation: The proposed method was validated for specificity, accuracy, and precision, the limit of detection, the limit of quantitation as well as the robustness of the method as per ICH guidelines. Replicate injections of the standard and sample were used to carry out all the studies.

Specificity: According to ICH Q2(R1), specificity is defined as the ability to assess the analyte unequivocally in the presence of components that may be expected to be present, which may be impurities and other products and was verified by injecting blank, standard, and sample and was found that no interference from the excipients of Formulation. Chromatograms of blank and placebo are exhibited in Fig. 6 and 7.

Linearity: Linearity was determined between the concentration ranges of $5-25 \mu \mathrm{g} / \mathrm{ml}$ for Sofosbuvir and $2-10 \mu \mathrm{g} / \mathrm{ml}$ for Ledipasvir. The injection was done twice for each concentration of Sofosbuvir and Ledipasvir. The correlation coefficient value was found to be 0.999 for Sofosbuvir and Ledipasvir. Linearity Results were shown in Table 1, and Calibration graphs were shown in Fig. 8 and 9. 


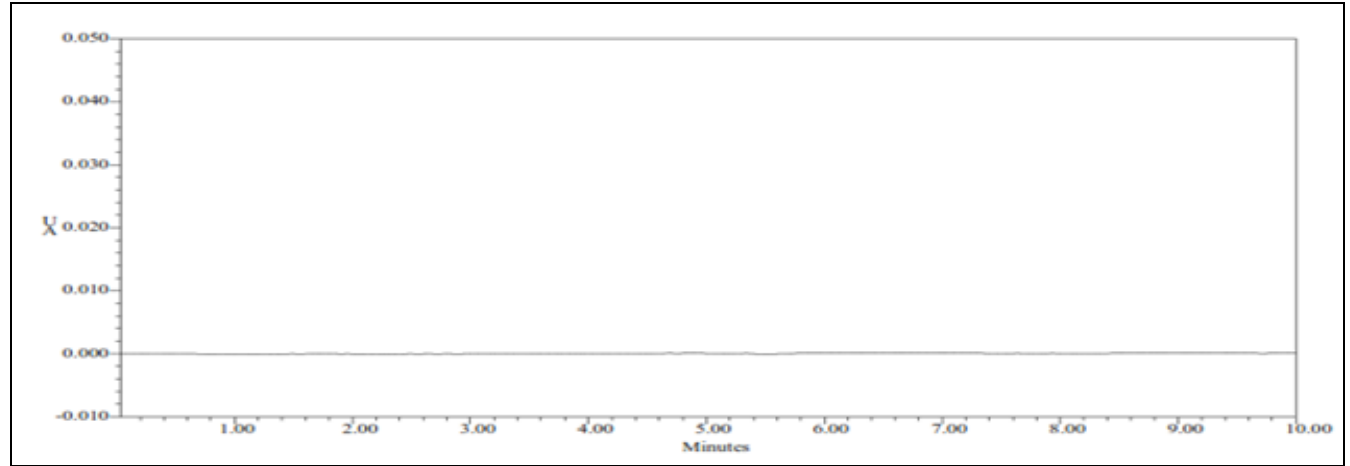

FIG. 6: CHROMATOGRAM OF BLANK

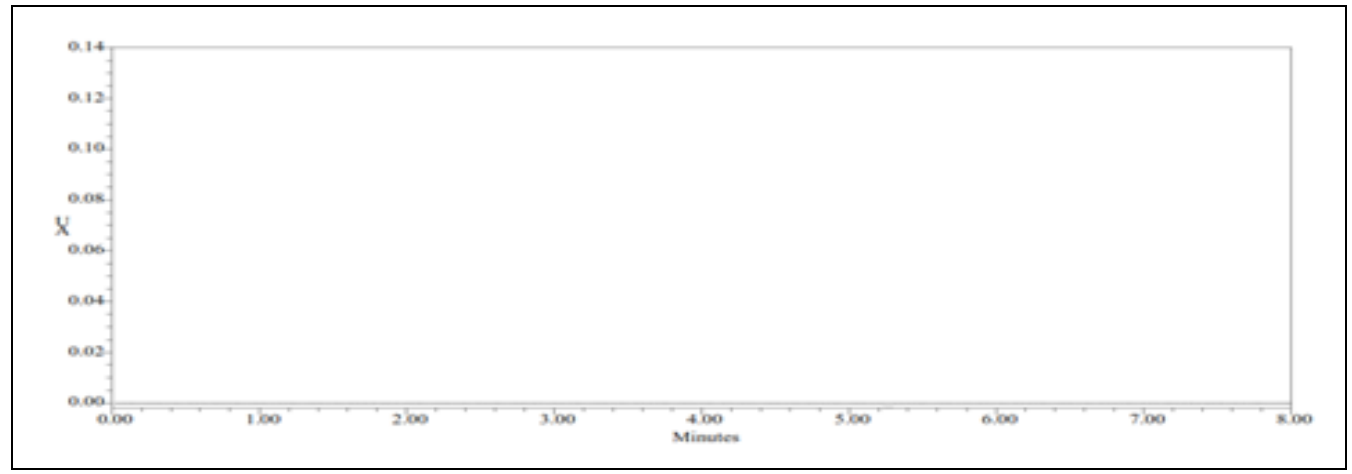

FIG. 7: CHROMATOGRAM OF PLACEBO

TABLE 1: LINEARITY VALUES OF SOFOSBUVIR AND LEDIPASVIR

\begin{tabular}{ccccc} 
S. no. & \multicolumn{2}{c}{ Sofosbuvir } & \multicolumn{2}{c}{ Ledipasvir } \\
\cline { 2 - 5 } & Concentration $(\boldsymbol{\mu g} / \mathbf{m l})$ & Area & Concentration $(\boldsymbol{\mu g} / \mathbf{m l})$ & Area \\
\hline 1 & 5 & 668029 & 2 & 293657 \\
2 & 10 & 1247781 & 4 & 557449 \\
3 & 15 & 1944421 & 6 & 798552 \\
4 & 20 & 2491191 & 8 & 1111601 \\
5 & 25 & 3230791 & 10 & 1395268 \\
\hline
\end{tabular}

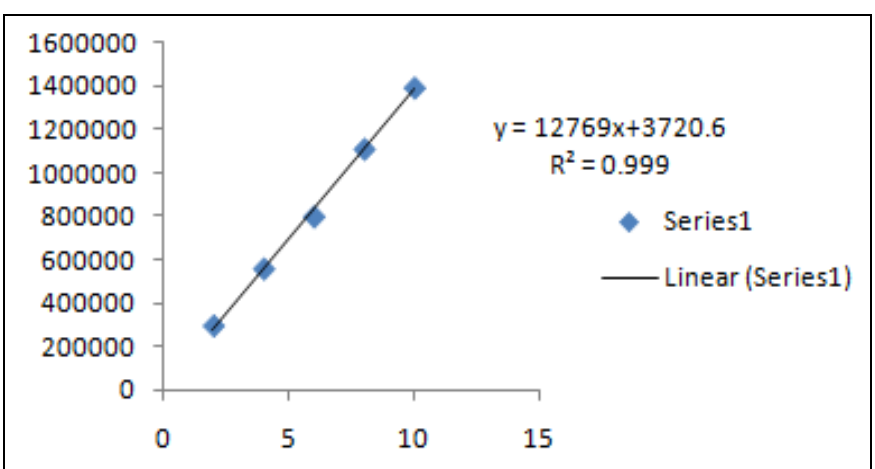

FIG. 8: CALIBRATION GRAPH OF SOFOSBUVIR

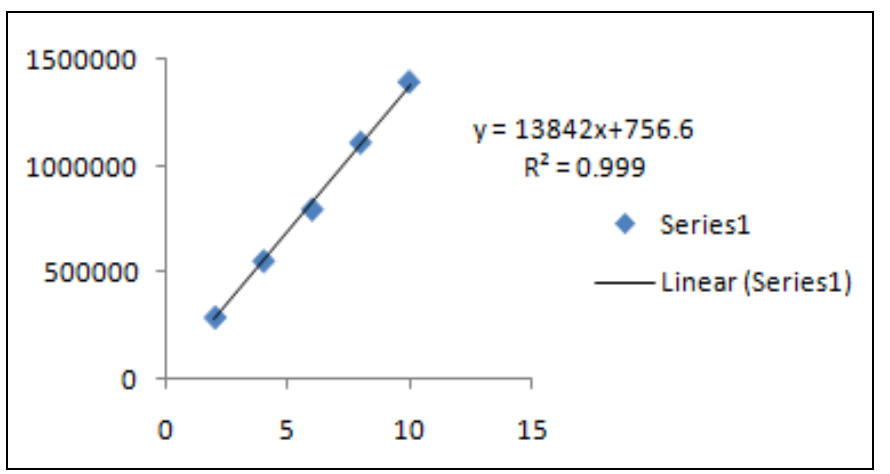

FIG. 9: CALIBRATION GRAPH OF LEDIPASVIR

TABLE 2: ACCURACY REPORT OF SOFOSBUVIR

\begin{tabular}{ccccc}
\hline$\%$ Level & $\begin{array}{c}\text { Amount Spiked } \\
(\boldsymbol{\mu g} / \mathbf{m L})\end{array}$ & $\begin{array}{c}\text { Amount recovered } \\
(\boldsymbol{\mu g} / \mathbf{m L})\end{array}$ & $\begin{array}{c}\% \\
\text { Recovery }\end{array}$ & $\begin{array}{c}\text { \% Mean } \\
\text { Recovery }\end{array}$ \\
\hline $50 \%$ & 7.5 & 7.39 & 98.55 & \\
& 7.5 & 7.40 & 98.72 & \\
$100 \%$ & 7.5 & 7.52 & 100.33 & \\
& 15 & 14.931 & 99.54 & \\
$150 \%$ & 15 & 15.018 & 100.12 & \\
& 15 & 15.076 & 90.51 & \\
& 22.5 & 22.108 & 98.26 & \\
\hline
\end{tabular}


Accuracy: The accuracy of an approach is the measurement of intimacy with respect to actuality worth for the sample and was determined by preparing concentration levels of 50\%, 100\%,
$150 \%$ and was injected thrice into the chromatographic system, and percentage recovery was calculated. The results are tabulated in Table 2 and Table 3.

TABLE 3: ACCURACY REPORT OF LEDIPASVIR

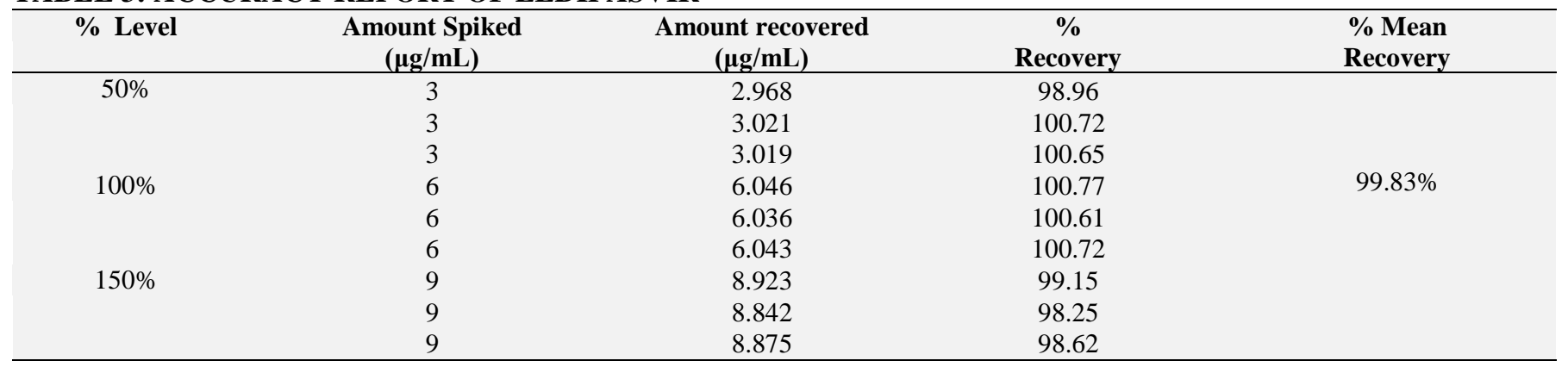

Precision: Precision of an analytical strategy was expressed by the closeness of agreement between a series of measurements obtained when multiple sampling of the homogenous sample under the prescribed conditions within the same day and inter-day. For precision, six repeated injections of standard and sample were made, and Percentage Relative Standard Deviation of each study was calculated and was found to be less than 2 showing the strategy was precise, and the results were shown in Table 4.

TABLE 4: PRECISION REPORT OF SOFOSBUVIR AND LEDIPASVIR

\begin{tabular}{ccc}
\hline S. no. & Area of Sofosbuvir & Area of Ledipasvir \\
\hline 1 & 1944421 & 798552 \\
2 & 1943452 & 798672 \\
3 & 1944521 & 799456 \\
4 & 1945397 & 798662 \\
5 & 1944425 & 798561 \\
6 & 1944731 & 798565 \\
Mean & 1944492 & 798745 \\
S.D & 7972.9 & 5034.7 \\
\%RSD & 0.3 & 0.4 \\
\hline
\end{tabular}

Limit of Detection and Limit of Quantitation: Limit of Detection is the lowest amount of analyte in a sample which can be detected but not quantitated, and Limit of Quantitation is the lowest amount of analyte in the sample that can be quantitatively determined and were calculated by using the formula $\mathrm{LOD}=3.3 \times \sigma / \mathrm{s}, \mathrm{LOQ}=10 \times$ $\sigma / \mathrm{S}$ Where, $\sigma=$ Standard deviation of the response $\mathrm{S}=$ Slope of the calibration curve. The results of LOD and LOQ were tabulated in Table 5.

TABLE 5: LOD \& LOQ VALUES OF SOFOSBUVIR AND LEDIPASVIR

\begin{tabular}{ccc}
\hline Compound & LOD & LOQ \\
\hline Sofosbuvir & 0.24 & 0.73 \\
Ledipasvir & 0.06 & 0.19 \\
\hline
\end{tabular}

Robustness: A robustness study was carried out by performing the flow rate variations from $0.9 \mathrm{~mL}$ $\mathrm{min}^{-1}$ to $1.1 \mathrm{~mL} \mathrm{~min}^{-1}$ and changes in mobile phase composition ranging from the more organic phase to less organic phase ratio. The proposed strategy was found to be robust only in less flow and also by a change in the composition of mobile phase $\pm 5 \%$. Ledipasvir and Sofosbuvir standard and samples were injected by changing chromatography conditions, and no significant difference in tailing factor and Plate Count was observed, and results are tabulated in Table 6.

TABLE 6: ROBUSTNESS REPORT OF SOFOSBUVIR AND LEDIPASVIR

\begin{tabular}{|c|c|c|c|}
\hline S. no. & Condition & $\begin{array}{c}\text { \% RSD of } \\
\text { Sofosbuvir }\end{array}$ & $\begin{array}{c}\text { \%RSD of } \\
\text { Ledipasvir }\end{array}$ \\
\hline 1 & $\begin{array}{c}\text { Flow rate (-) } \\
0.9 \mathrm{ml} / \mathrm{min}\end{array}$ & 0.4 & 0.5 \\
\hline 2 & $\begin{array}{c}\text { Flow rate }(+) \\
1.1 \mathrm{ml} / \mathrm{min}\end{array}$ & 0.6 & 1.1 \\
\hline 3 & Mobile phase (-) & 0.4 & 1.8 \\
\hline 4 & Mobile phase (+) & 0.4 & 0.3 \\
\hline 5 & Temperature (-) & 0.5 & 0.2 \\
\hline 6 & Temperature (+) & 0.4 & 0.4 \\
\hline
\end{tabular}

Recovery Studies: Standard addition method is performed at 50,100,150\% levels for Ledipasvir and Sofosbuvir, and interference of formulation additives was tested. The Recovery was calculated based on the amount of drug found and was found to be in the range of $98-102 \%$.

CONCLUSION: The Validated Chromatographic Strategy was found to be accurate, simple, and decisive for the quantitative estimation of Ledipasvir and Sofosbuvir in bulk and fixed dosage form. Different trials were carried out to determine the optimized chromatographic conditions, and an 
initial attempt was performed by utilizing a low proportion of organic solvents for the elution of compounds by reducing the retention time of the compounds, which made the strategy economical. The proposed method is easy, speedy, and measurably substantial. During the drug analysis, no interfering peak was found within the chromatogram, indicating that there is no excipient interference. Hence this method can be employed for routine quality control analysis of Ledipasvir and Sofosbuvir samples.

ACKNOWLEDGEMENT: Authors are very thankful to Nutech Biosciences Pvt., Ltd., for providing the required facilities to carry out the research work.

CONFLICTS OF INTEREST: The authors declare that they have no conflict of interest.

\section{REFERENCES:}

1. Rathod SM and Patel PU: Development and validation for simultaneous estimation of Sofosbuvir and Daclatasvir dihydrochloride in pharmaceutical dosage form by ratio derivative and dual wavelength methods. International Journal of Pharmac Quality Assurance 2020; 11(1): 25-31.

2. Rani JS and Devanna N: Development and validation of RP-HPLC method for the simultaneous estimation of sofosbuvir, velpatasvir and voxilaprevir in bulk and tablet dosage forms. Rasāyan Journal of Chemistry 2018; 11: 452-59.

3. Hemdan A and Eissa MS: Simultaneous chromatographic analysis of Sofosbuvir/Ledipasvir in their combined dosage form: an application to green analytical chemistry. J of Anal Science and Technology 2019; 10(39): 1-12.

4. Baker MM, El-Kafrawy DS, Mahrous MS and Belal TS: Validated spectrophotometric and chromatographic methods for analysis of the recently approved hepatitis C antiviral combination ledipasvir and sofosbuvir. Annales Pharmaceutiques Françaises Journal 2018; 76(1): 16-31.
5. Rote AP, Alhat $\mathbf{J}$ and Kulkarni AA: Development and validation of RP-HPLC method for the simultaneous estimation of ledipasvir and sofosbuvir in bulk and pharmaceutical dosage form. International Journal of Pharmaceutical Sciences and Drug Res 2017; 9(6): 291-98.

6. Bandla $\mathbf{J}$ and Ganapat $S$ : Development and validation of a stability-indicating method for the simultaneous estimation of sofosbuvir and ledipasvir by RP-HPLC. Indian Journal of Pharmaceutical Sciences 2018; 80(6): 1170-76.

7. Mastanamma SK, Chandini SK, Reehana SK and Saidulu P: Development and validation of stability indicating RPHPLC method for the simultaneous estimation of Sofosbuvir and Ledipasvir in bulk and their combined dosage form. Future Journal of Pharmaceutical Sciences 2018; 4(2): 116-123.

8. Khalili M, Sohrabi MR, Mirzabeygi V and Ziaratgahi TN: Chemometric simultaneous determination of sofosbuvir and ledipasvir in pharmaceutical dosage form. Spectrochimica acta Part A, Molecular and Biomolecular Spectroscopy 2018; 194: 141-51.

9. Abdulkareem A and El-Tohamy MF: Validated capillary zone electrophoresis approach for simultaneous separation and determination of hepatitis $\mathrm{C}$ sofosbuvir and ledipasvir in tablet dosage form. World Journal of Pharmaceutical Research 2017; 6(5): 129-47.

10. Devi LM, Reddy TR and Abbul K: Simultaneous determination and validation of third generation antiviral drugs by RP-HPLC method. International journal of pharmaceutical analysis and Research 2019; 8: 1-8.

11. Kothapalli KK, Saisri M, Priyanka M, Subhashini $M$ and Manikanta M: A New Analytical Method Development and Validation for the Simultaneous Estimation of Ledipasvir and Sofosbuvir Using RP-HPLC. Intercontinental Journal of Pharmaceutical Investigation and Research 2017; 4(1):142-165.

12. Revathi K, Sowndarya G and Swathi V: New RP-HPLC method for simultaneous estimation of sofosbuvir and velpatasvir in combined pharmaceutical dosage forms. International Journal of Advances in Pharmacy and Biotechnology 2020; 6(3): 19-22.

13. EL-Shorbagy HI, Elsebaei F, Hammad SF, Amina M and El-Brashy: Optimization and modeling of a green dual detected RP-HPLC method by UV and fluorescence detectors using two level full factorial design for simultaneous determination of sofosbuvir and ledipasvir: Application to average content and uniformity of dosage unit testing. Microchemical Journal 2019; 147: 374-92.

How to cite this article:

Kumar VP, Harikrishnan N and Subramanian G: RP-HPLC method development and validation for the simultaneous estimation of ledipasvir and sofosbuvir in fixed dosage form. Int J Pharm Sci \& Res 2021; 12(7): 3852-57. doi: 10.13040/IJPSR.0975-8232.12(7).3852-57.

All @ 2013 are reserved by the International Journal of Pharmaceutical Sciences and Research. This Journal licensed under a Creative Commons Attribution-NonCommercial-ShareAlike 3.0 Unported License.

This article can be downloaded to Android OS based mobile. Scan QR Code using Code/Bar Scanner from your mobile. (Scanners are available on Google Playstore) 